\title{
Does size matter? Addressing pack size and antibiotic duration
}

\section{Treasure M McGuire \\ Assistant director of pharmacy, Mater Health \\ Services, Brisbane \\ Conjoint senior lecturer, \\ School of Pharmacy, \\ University of Queensland, \\ Brisbane \\ Associate professor of pharmacology, Faculty of Health Sciences and Medicine, Bond University, Gold Coast, Queensland}

\section{Keywords}

antibiotic resistance, clinical practice guidelines, drug packaging

Aust Prescr 2019;42:2-3 https://doi.org/10.18773/ austprescr.2019.005
In Australia, most antibiotics are prescriptiononly so prescribers are the custodians of judicious use. They need to balance their concerns about antibiotic resistance with their responsibility for individual patient management. Prescribing with no clinical indication, inappropriate drug choice, and suboptimal dosing and duration can all contribute to antimicrobial resistance., ${ }^{1,2}$ Clinical practice guidelines are therefore important for improving the quality and cost-effectiveness of infectious disease management. However, subtle factors such as the size of antibiotic packs could impact on judicious antibiotic use.

Antibiotic prescribing in primary care is largely empiric and symptom based. Antibiotics are usually started without microbiological testing. Clinical practice guidelines usually focus on antibiotic choice, with a recommended dose, frequency and duration, but have limited advice on resistance patterns. ${ }^{3}$ Optimising the dose and duration of antibiotic treatment could simultaneously minimise both the symptomatic period and the selection pressure for resistance. ${ }^{4}$

Clinical practice guideline recommendations differ globally, particularly for the duration of antibiotic therapy. ${ }^{5,6}$ When two UK guidelines for communityacquired pneumonia were critically assessed, there was a key difference of 2-3 days in the recommended course of antibiotics for infections of low-moderate severity. ${ }^{5}$ Similarly, there were major differences in the recommended treatment duration for paediatric infections across seven reputable clinical practice guidelines. ${ }^{6}$ The fact that recommendations about the duration of therapy are based more on expert opinion than strong scientific evidence is not widely appreciated by clinicians.

It is clear that no 'one-size-fits-all' for the length of an antibiotic course. ${ }^{4}$ Infection resolution requires the antimicrobial to reach and remain at the site of infection in a sufficient concentration for a sufficient time. Concentration-dependent antibiotics such as aminoglycosides display maximal bactericidal activity at high concentrations, even if these concentrations are maintained for a relatively short time. In contrast, antibiotics displaying timedependent activity, for example beta-lactams, require free drug at the infection site to be above the minimum inhibitory concentration or breakpoint concentration for a longer time. Duration can therefore be influenced by not only the dose prescribed but also the inherent characteristics of the antibiotic. ${ }^{\text {? }}$

Judicious antibiotic use needs to balance prescribing for too short a period (causing treatment failure, delayed return to health or the development of complications) with overprescribing which increases the risk of resistance, non-adherence, adverse effects and cost. Sub-therapeutic antibiotic concentrations can encourage antibiotic-resistant bacteria..$^{8}$ Other considerations when prescribing include the characteristics of the infecting organism, the patient's immune status and the bacterial gene pool.

While clinical evidence favours prolonged treatment to prevent the relapse of conditions such as enterococcal endocarditis, only short courses are needed for uncomplicated urinary tract infections in women. Evaluation of 13 meta-analyses to optimise antimicrobial duration in common bacterial infections determined that the duration of therapy could be shortened in most of these infections by at least three days without compromising patient outcomes. ${ }^{9}$ However, for many infections managed in the community, the optimum treatment duration is unknown.

To improve the likelihood of success in clinical trials, a longer duration of antibiotics than the theoretical minimum may be used. Only after establishing efficacy are equivalence trials of shorter durations conducted. As non-inferiority trials require large numbers of patients, cost drives trial design towards single rather than multiple duration arms. Several pharmacokinetic and pharmacodynamic models have been proposed for duration-randomised trials to overcome cost as a barrier. ${ }^{10}$ To extend the lifespan of antibiotics there needs to be collaboration between researchers, clinicians and the pharmaceutical industry to conduct equivalence trials. These are needed to determine the optimal minimum antibiotic regimen for common infections in Australia.

Ambiguity about the optimal duration of treatment for a particular indication contributes to uncertainty about how many doses to put in a pack. However, pack size heavily influences the duration of use. It will continue to do so while consumers are given advice to 'complete the antibiotic course'.

A 2015 analysis of published data on the most commonly prescribed antibiotics in Australian primary 
care and their most common indications found a clear mismatch between the recommended treatment duration in clinical practice guidelines and the pharmaceutical industry packaging. ${ }^{11}$ Of 32 common prescribing scenarios, 10 had doses in surplus and 18 had a shortfall, leaving only four where the pack size matched the recommended duration. In only two cases was a shortfall addressed by a repeat prescription.

Any mismatch between pack size and doses consumed might contribute to leftover antibiotics in the community. If these antibiotics are subsequently taken by the patient or someone else, it would contribute to potentially inappropriate use and, thereby, resistance. Alternatively, unused antibiotics could be discarded into the environment (landfill or waste water) which may facilitate the development or proliferation of resistant strains of bacteria. ${ }^{12}$

While solutions are not obvious, we must be willing to try strategies to reduce the mismatch between guidelines and antibiotic packaging. Regulations could require the industry to package antibiotics in accordance with clinical practice guidelines. While multiple pack sizes would increase costs, government incentives for the production of small packs could increase dispensing flexibility and minimise waste.
Prescribing software could improve adherence to clinical practice guidelines by commencing with the intended indication instead of the antibiotic. The indication would activate consensus regimens supported by evidence (or lack thereof). Prescriptions would not be printed until dose, frequency and importantly duration were entered, overriding the default pack and calculating the required quantity. Pharmacists would require corresponding dispensing software. They would also need to spend more time to implement safety strategies to prevent the reuse of broken packs with varying expiry dates.

Clinicians and the public should be informed that completing the pack is no longer supported by evidence and that resistance is primarily due to overuse. Patients should be empowered to stop their antibiotic after a specified minimum number of days or when they feel better (whichever comes first) and to return any unused doses for safe disposal to the pharmacy where the medicine was dispensed. ${ }^{13}$ These and similar strategies warrant discussion to potentially extend the lifespan of antibiotics without compromising patient care. $<$

Conflict of interest: none declared

\section{REFERENCES}

1. DeRyke CA, Lee SY, Kuti JL, Nicolau DP. Optimising dosing strategies of antibacterials utilising pharmacodynamic principles: impact on the development of resistance. Drugs 2006;66:1-14. https://doi.org/10.2165/ 00003495-200666010-00001

2. Costelloe C, Metcalfe C, Lovering A, Mant D, Hay AD. Effect of antibiotic prescribing in primary care on antimicrobial resistance in individual patients: systematic review and meta-analysis. BMJ 2010;340:c2096. https://doi.org/10.1136/ bmj.c2096

3. Elias C, Moja L, Mertz D, Loeb M, Forte G, Magrini N. Guideline recommendations and antimicrobial resistance: the need for a change. BMJ Open 2017;7:e016264. https://doi.org/10.1136/bmjopen-2017-016264

4. Geli P, Laxminarayan R, Dunne M, Smith DL. "One-size-fitsall"? Optimizing treatment duration for bacterial infections. PLoS One 2012;7:e29838. https://doi.org/10.1371/ journal.pone.0029838

5. Lim WS, Smith DL, Wise MP, Welham SA; British Thoracic Society. British Thoracic Society community acquired pneumonia guideline and the NICE pneumonia guideline: how they fit together. Thorax 2015:70:698-700. https://doi.org/10.1136/thoraxjnl-2015-206881

6. Kerrison C, Riordan FA. How long should we treat this infection for? Arch Dis Child Educ Pract Ed 2013:98:136-40. https://doi.org/10.1136/archdischild-2013-304135
7. Adembri C, Novelli A. Pharmacokinetic and pharmacodynamic parameters of antimicrobials: potentia for providing dosing regimens that are less vulnerable to resistance. Clin Pharmacokinet 2009;48:517-28. https://doi.org/10.2165/10895960-000000000-00000

8. Rybak MJ. Pharmacodynamics: relation to antimicrobial resistance. Am J Infect Control 2006;34(Suppl 1):S38-45. https://doi.org/10.1016/j.ajic.2006.05.227

9. Rafailidis PI, Pitsounis Al, Falagas ME. Meta-analyses on the optimization of the duration of antimicrobial treatment for various infections. Infect Dis Clin North Am 2009;23:269-76. https://doi.org/10.1016/j.idc.2009.01.009

10. Horsburgh CR, Shea KM, Phillips P, Lavalley M. Randomized clinical trials to identify optimal antibiotic treatment duration. Trials 2013;14:88. https://doi.org/10.1186/1745-6215-14-88

11. McGuire TM, Smith J, Del Mar C. The match between common antibiotics packaging and guidelines for their use in Australia. Aust N Z J Public Health 2015;39:569-72. https://doi.org/10.1111/1753-6405.12385

12. Chow L, Waldron L, Gillings MR. Potential impacts of aquatic pollutants: sub-clinical antibiotic concentrations induce genome changes and promote antibiotic resistance. Front Microbiol 2015;6:803. https://doi.org/10.3389/ fmicb.2015.00803

13. Llewelyn MJ, Fitzpatrick JM, Darwin E, Tonkin-Crine S, Gorton C, Paul J, et al. The antibiotic course has had its day. BMJ 2017;358:j3418. https://doi.org/10.1136/bmj.j3418

\section{FURTHER READING}

Dyar OJ, Beović B, Vlahović-Palčevski V, Verheij T, Pulcini C; on behalf of ESGAP (the ESCMID [European Society of Clinical Microbiology and Infectious Diseases] Study Group for Antibiotic Policies). How can we improve antibiotic prescribing in primary care? Expert Rev Anti Infect Ther 2016;14:403-13. https://doi.org/ 10.1586/14787210.2016.1151353

Finch RG, Low DE. A critical assessment of published guidelines and other decision-support systems for the antibiotic treatment of community-acquired respiratory tract infections. Clin Microbiol Infect 2002;8 Suppl 2:69-91. https://doi.org/ 10.1046/j.1469-0691.8.s.2.7.x
O'Neill J, chair. Tackling drug-resistant infections globally: final report and recommendations. The review on antimicrobial resistance. London: Wellcome Trust and UK Government. May 2016. https://amr-review.org [cited 2019 Jan 3]. 